\title{
Platelet-lymphocyte ratios: a potential marker for pulmonary tuberculosis diagnosis in COPD patients
}

This article was published in the following Dove Press journal: International Journal of COPD

3 November 2016

Number of times this article has been viewed

\section{Guozhong Chen \\ Chunling Wu \\ Zhiying Luo \\ Yiming Teng \\ Suping Mao}

Department of Respiratory Medicine, Yiwu Central Hospital, Yiwu, Zhejiang Province, People's Republic of China

Correspondence: Guozhong Chen Department of Respiratory Medicine, Yiwu Central Hospital, No 699 Jiangdong Road, Yiwu City, 322000 Zhejiang Province, People's Republic of China Tel +865798520 987l

Fax +86 5798520 987।

Email guozhongchen20I5@sina.com
Background: In recent decades, morbidity and mortality have been found to be significantly increased in patients with chronic obstructive pulmonary disease (COPD) complicated with pulmonary tuberculosis (PTB). Platelet-lymphocyte ratio (PLR) is an indicator for inflammatory diseases. This study aims to investigate whether PLR could act as a potential marker for patients with COPD complicated with PTB.

Methods: In this retrospective study, laboratory characteristics of 87 COPD patients complicated with PTB (determined by Mycobacterium tuberculosis positive culture from sputum or bronchial lavage fluid) and 83 COPD patients (as the control group, determined by M. tuberculosis culture negativity from sputum or bronchial lavage fluid) were investigated. Data obtained on the day of admission were analyzed.

Results: PLR $>216.82$ was identified as the optimal cutoff value for discriminating COPD patients with PTB (sensitivity $92.4 \%$, specificity $84.5 \%$, positive-predictive value $91.6 \%$, negative-predictive value $86.2 \%$, and area under the curve [AUC] was 0.87 ) from patients with COPD alone. The AUC of PLR was significantly greater than that of neutrophil-lymphocyte count ratio (AUC, 0.74; 95\% confidence interval, 0.67-0.81; $P<0.01$ ).

Conclusion: PLR could be developed as a valuable maker for identifying tuberculosis infection in COPD patients.

Keywords: platelet to lymphocyte ratio, pulmonary tuberculosis, chronic obstructive pulmonary disease

\section{Introduction}

Pulmonary tuberculosis (PTB), a common infectious disease caused by Mycobacterium tuberculosis $(\mathrm{Mtb})$, is an important public health problem. ${ }^{1}$ In recent decades, PTB has begun to rebound quickly worldwide, and the incidence of PTB in developing countries is also relatively high compared to that in developed countries. ${ }^{1}$ Chronic obstructive pulmonary disease (COPD) is a chronic airway inflammatory disease with a high prevalence rate worldwide and is one of the main causes of morbidity and mortality globally. ${ }^{2}$ PTB could be a frequent comorbid condition in patients with COPD, which may be due to smoking and use of corticosteroids. ${ }^{3-5}$ COPD was identified as an independent risk factor for PTB. ${ }^{4,6}$ COPD complicated with PTB is a dangerous combination. Also, the delayed diagnosis of PTB in COPD patients could contribute to prevent further disease progression, increase mortality, and transmission of PTB within the community, which could be an important public health issue. ${ }^{7}$ Lee et al suggested that it is necessary to keep a high suspicion and regularly monitor for the development of PTB in COPD patients. ${ }^{4}$ 
There are several diagnostic approaches for PTB diagnosis, such as clinical features, radiological examination, and laboratory examination. ${ }^{8}$ However, when PTB occurs in COPD patients, it is difficult to identify the condition at an early stage, as symptoms of PTB, such as cough and fever, could also be seen in COPD patients. Meanwhile, it is difficult to get typical radiological findings of PTB at an early stage. It has been suggested that the only definitive diagnostic method known for PTB is smear positivity or the growth of Mtb bacilli from pulmonary materials such as sputum or bronchoscopic lavage fluid. Nevertheless, it cannot be routinely performed in COPD patients, as the growth of Mtb bacilli is a specific diagnostic technology for patients suspected of having tuberculosis (TB) infection. Therefore, it is desirable to find an indicator for PTB, which may provide guidance at the diagnostic stage and could be obtained from routine examination in COPD patients.

Inflammatory markers investigated in routine blood examination are extensively used as factors for differential diagnosis and prognostic evaluation in several situations such as inflammatory diseases, cancer, and cardiovascular disease. ${ }^{9-14}$ Evidences have shown that neutrophil-lymphocyte count ratio (NLR) could be used as an effective indicator for differential diagnosis between PTB and bacterial community-acquired pneumonia (CAP), ${ }^{9}$ or sarcoidosis. ${ }^{10}$ The importance of platelet-lymphocyte ratio (PLR) has been emphasized as a marker in some disorders such as non-small-cell lung cancer, acute coronary syndrome, end-stage renal disease, and so on. ${ }^{11-13}$ However, whether PLR, a readily obtainable positive inflammation marker, could act as a potential indicator for PTB in COPD patients is unclear. This study aims to investigate the usability of the PLR for differential diagnosis between COPD patients complicated with PTB and COPD patients.

\section{Materials and methods}

The study has been approved by the institutional review board of Yiwu Central Hospital, Yiwu, People's Republic of China. As this is a retrospective study using an encrypted database, the institutional review board waived the need for informed consent for this study. Patient records/information was anonymized and deidentified prior to analysis.

Patients from the Yiwu Central Hospital who were older than 18 years and were diagnosed as COPD (control group) ${ }^{15}$ or COPD complicated with PTB (PTB group) from January 2010 to December 2013 were included in this retrospective cohort study. Patients with COPD and showing growth of Mtb bacilli in pulmonary material were eligible for the PTB group. COPD patients with culture negative for Mtb were eligible for the control group.
In the present study, information such as patients' age, sex, erythrocyte sedimentation rate (ESR), C-reactive protein (CRP), and complete blood count were collected retrospectively based on hospital records on the day of admission. The NLR or PLR was obtained by dividing the absolute number of neutrophils or platelets by lymphocytes in complete blood count, respectively. The complete blood count was measured by an automatic blood count device.

Exclusion criteria were as follows: presence of hematologic disorders, peripheral vascular disease; currently under steroid therapy (including the inhaled steroids), nonsteroidal anti-inflammatory drugs, anticoagulant medications; history of chemotherapy or radiotherapy within 4 weeks before enrollment; absence of Mtb pulmonary material culture, ESR, or CRP data.

\section{Statistical analysis}

All statistical analyses were performed using Statistical Package for the Social Sciences 17.0 (SPSS Inc., Chicago, IL, USA). Continuous data are presented as the mean \pm standard deviation. Continuous variables were compared by Student's $t$-test, and the differences between continuous variables with non-normal distribution were estimated with MannWhitney test. Categorical data are presented as frequencies. Differences between categorical variables were analyzed using Pearson's chi-square test or Fisher's exact test. Receiver operating characteristic curve analysis was performed to identify the optimal cutoff levels for NLR and PLR, and to identify the greatest sum of sensitivity and specificity for distinguishing PTB group from the control group. The ability of NLR and PLR to distinguish the PTB group from the control group was compared using the area under the curve (AUC). A $P$-value $\leq 0.05$ was considered statistically significant.

\section{Results}

A total of 170 patients who fulfilled the inclusion criteria were enrolled in the present study. The PTB group consisted of 87 patients, and the control group had 83 patients. The median age of patients in the PTB and control groups were 49 and 51 years, respectively $(P=0.16)$ (Table 1$)$. Also, $57 \%$ of patients were male and $36 \%$ were female in the control and PTB groups, respectively $(P=0.67)$ (Table 1).

A statistically significant difference was found between the groups in terms of ESR, CRP, NLR, and PLR $(P<0.01)$ (Table 1), when the PTB and control groups were compared with each other.

A receiver operating characteristic curve was generated to determine the sensitivity and specificity of NLR, CRP, 
Table I Demographic and laboratory characteristics of the population

\begin{tabular}{llll}
\hline Characteristics & Control & PTB & P-value \\
\hline Age (years) & $5 \mathrm{I}(46-7 \mathrm{I})$ & $49(52-66)$ & 0.16 \\
Sex (male/female) & $48 / 35$ & $56 / 3 \mathrm{I}$ & 0.67 \\
ESR (mm/st) & 16 & 57 & $<0.0 \mathrm{I}$ \\
CRP $(\mathrm{mg} / \mathrm{L})$ & $3.12 \pm 0.08$ & $84.4 \mathrm{I} \pm 0.76$ & $<0.0 \mathrm{I}$ \\
NLR & $\mathrm{I} .94 \pm 0.0 \mathrm{I}$ & $4.47 \pm 0.12$ & $<0.0 \mathrm{I}$ \\
PLR & $\mathrm{I} 30.2 \mathrm{I} \pm 45.30$ & $287.05 \pm 32.76$ & $<0.0 \mathrm{I}$
\end{tabular}

Note: Data presented as mean (range), mean \pm standard deviation, or $\mathrm{n}$. Abbreviations: CRP, C-reactive protein; ESR, erythrocyte sedimentation rate; NLR, neutrophil to lymphocyte ratio; PLR, platelet-lymphocyte ratio; PTB, pulmonary tuberculosis.

ESR, and PLR. An NLR $>2.67$ was identified as the optimal cutoff value for discriminating PTB from COPD patients, yielding $61 \%$ sensitivity, $68 \%$ specificity, $72 \%$ positivepredictive value, and $63 \%$ negative-predictive value. Based on this cutoff value, accuracy of the NLR test for distinguishing TB group from the control group was $74 \%$. The NLR AUC $(0.74 ; 95 \%$ confidence interval [CI], 0.67-0.81) was significantly greater than that of CRP (AUC, $0.61 ; 95 \%$ CI, $0.56-0.69 ; P<0.05)$ and ESR (AUC, $0.54 ; 95 \% \mathrm{CI}$, $0.45-0.60 ; P<0.05)$.

PLR $>216.82$ is the optimal cutoff value for discriminating PTB from the control group, yielding 92.4\% sensitivity, $84.5 \%$ specificity, $91.6 \%$ positive-predictive value, and $86.2 \%$ negative-predictive value. Based on this cutoff value, accuracy of the PLR test for distinguishing TB group from the control group was $82 \%$. The PLR AUC (AUC, 0.87; $95 \% \mathrm{CI}, 0.73-0.91 ; P<0.05)$ was significantly greater than that of NLR.

\section{Discussion}

Inflammation plays an important role in the immune reaction against PTB. However, cellular immunity was impaired in COPD patients complicated with PTB. ${ }^{16}$ The present study demonstrates that NLR and PLR, which reflect the ratio of cells in innate immunity, could be used as markers for differential diagnosis between COPD patients complicated with PTB and COPD patients.

NLR has been identified as an indicator for inflammatory diseases. ${ }^{17}$ Recent studies have shown that NLR is a new marker of PTB..$^{910}$ Iliaz et al indicated that NLR is a useful marker for differentiating TB and sarcoidosis. ${ }^{10}$ Also, NLR plays a valuable role in the differential diagnosis between PTB and bacterial CAP. ${ }^{9}$ The value of NLR was markedly higher in advanced PTB compared to that in mild to moderate PTB. ${ }^{18}$ In the present study, NLR played a useful role in the differential diagnosis between COPD patients complicated with PTB and COPD patients. Based on previous studies, ${ }^{11-13}$ an NLR of 2.67 was assigned as the optimal cutoff value in this study to differentiate between comorbid patients and patients with COPD alone. Actually, NLR of 2.67 has been become a gold standard in clinical practice. Therefore, in this study, an NLR $>2.67$ was identified as the optimal cutoff value for discriminating PTB from COPD patients. It is similar to the value in the differential diagnosis of sarcoidosis and $\mathrm{TB}$, but far from the value in the differential diagnosis of PTB and CAP. This result may due to the significantly increased neutrophil levels in the CAP, as the neutrophil levels in PTB, COPD, and sarcoidosis were increased mildly compared with the levels in CAP patients. Based on a previous study, ${ }^{19}$ a CRP level of $7 \mathrm{mg} / \mathrm{L}$ and ESR of $12 \mathrm{mg} / \mathrm{L}$ were assigned as the optimal cutoff values to differentiate between comorbid patients and patients with COPD alone. In our study, the AUC of ESR or CRP was lower than NLR. These results indicate that NLR may be a marker for the differential diagnosis between COPD patients complicated with PTB and patients with COPD alone.

PLR has also been known as a marker for several diseases such as cancer, cardiovascular disease, and inflammatory diseases, and PLR $>216.82$ is the optimal cutoff value for discriminating PTB group from the control group. ${ }^{11-13,20}$ Therefore, we selected PLR $>216.82$ as the gold standard for the optimal cutoff value in this study. The pretreatment NLR and PLR represented significant prognostic indicators of survival in early-stage non-small-cell lung carcinoma patients treated with stereotactic radiation. ${ }^{11}$ Akkaya et al found that PLR is a convenient, easily measured prognostic indicator for patients with acute coronary syndrome. ${ }^{12}$ It is suggested that PLR is a better indicator for inflammation in end-stage renal disease patients, compared with NLR. ${ }^{13}$ In the present study, the PLR AUC was significantly greater than that of NLR. The results indicate that the PLR could be a potential marker for identifying PTB in COPD patients. PLR can be obtained from a routine blood examination on the day of admission. Therefore, it can provide an opportunity to distinguish PTB at an early stage and to give timely treatment with anti-TB agents. Previous studies reported that sensitivity more than $90 \%$, specificity more than $80 \%$, positive-predictive value more than $90 \%$, and negative-predictive value more than $80 \%$ are considered as the gold standard in the biomarkers for diagnosis. ${ }^{21-23} \mathrm{We}$ suggest that PLR is a useful and easily obtainable marker for identifying PTB in COPD patients.

Although we obtained some interesting findings, there were also some limitations in this study. Firstly, the patient sample size included in this study is relatively small for exploring the biomarker of disease diagnosis. Secondly, we did not investigate the other biomarkers for PTB in COPD 
patients in this study. Thirdly, the sample size of the COPD patients was not calculated in this study.

In conclusion, the present study suggests that both NLR and PLR reflect the ratio of cells in innate immunity, and both could be used as the markers for differential diagnosis between COPD patients complicated with PTB and patients with COPD alone. However, the AUC of PLR was significantly greater than that of NLR. Therefore, we conclude that PLR could be developed as a valuable maker for identifying $\mathrm{TB}$ infection in COPD patients.

\section{Acknowledgment}

The authors wish to thank Great Duo for the useful comments.

\section{Disclosure}

The authors report no conflicts of interest in this work.

\section{References}

1. Reves R, Schluger NW. Update in tuberculosis and nontuberculous mycobacterial infections 2013. Am J Respir Crit Care Med. 2014; 189(8):894-898.

2. Vestbo J, Hurd SS, Agustí AG, et al. Global strategy for the diagnosis, management, and prevention of chronic obstructive pulmonary disease: GOLD executive summary. Am J Respir Crit Care Med. 2013; 187(4):347-365.

3. Dong YH, Chang CH, Lin Wu FL, et al. Use of inhaled corticosteroids in patients with COPD and the risk of TB and influenza: a systematic review and meta-analysis of randomized controlled trials. a systematic review and meta-analysis of randomized controlled trials. Chest. 2014;145(6):1286-1297.

4. Lee CH, Lee MC, Shu CC, et al. Risk factors for pulmonary tuberculosis in patients with chronic obstructive airway disease in Taiwan: a nationwide cohort study. BMC Infect Dis. 2013;13:194.

5. Kim JH, Park JS, Kim KH, Jeong HC, Kim EK, Lee JH. Inhaled corticosteroid is associated with an increased risk of TB in patients with COPD. Chest. 2013;143(4):1018-1024.

6. Inghammar M, Ekbom A, Engström G, et al. COPD and the risk of tuberculosis - a population-based cohort study. PLoS One. 2010;5(4): e10138.

7. Pablos-Méndez A, Sterling TR, Frieden TR. The relationship between delayed or incomplete treatment and all-cause mortality in patients with tuberculosis. JAMA. 1996;276(15):1223-1228.

8. Curley CA. Rule out pulmonary tuberculosis: clinical and radiographic clues for the internist. Cleve Clin J Med. 2015;82(1):32-38.
9. Yoon NB, Son C, Um SJ. Role of the neutrophil-lymphocyte count ratio in the differential diagnosis between pulmonary tuberculosis and bacterial community-acquired pneumonia. Ann Lab Med. 2013; 33(2):105-110.

10. Iliaz S, Iliaz R, Ortakoylu G, Bahadir A, Bagci BA, Caglar E. Value of neutrophil/lymphocyte ratio in the differential diagnosis of sarcoidosis and tuberculosis. Ann Thorac Med. 2014;9(4):232-235.

11. Cannon NA, Meyer J, Iyengar P, et al. Neutrophil-lymphocyte and platelet-lymphocyte ratios as prognostic factors following stereotactic radiation therapy for early-stage non-small cell lung cancer. $J$ Thorac Oncol. 2015;10(2):280-285.

12. Akkaya E, Gul M, Ugur M. Platelet to lymphocyte ratio: a simple and valuable prognostic marker for acute coronary syndrome. Int J Cardiol. 2014;177(2):597-598.

13. Turkmen K, Erdur FM, Ozcicek F, et al. Platelet-to-lymphocyte ratio better predicts inflammation than neutrophil-to-lymphocyte ratio in end-stage renal disease patients. Hemodial Int. 2013;17(3):391-396.

14. Arbel Y, Shacham Y, Ziv-Baran T, et al. Higher neutrophil/lymphocyte ratio is related to lower ejection fraction and higher long-term all-cause mortality in ST-elevation myocardial infarction patients. Can J Cardiol. 2014;30(10):1177-1182.

15. Global Initiative for Chronic Obstructive Lung Disease. Global strategy for the diagnosis, management and prevention of chronic obstructive pulmonary disease (Updated 2014). Available from: http://www. goldcopd.org/uploads/users/files/GOLD_Report_2014_Jun11.pdf. Accessed August 16, 2014.

16. Tang S1, Cui H, Yao L, et al. Increased cytokines response in patients with tuberculosis complicated with chronic obstructive pulmonary disease. PLoS One. 2013;8(4):e62385.

17. Günay E, Sarınç Ulaşlı S, Akar O, et al. Neutrophil-to-lymphocyte ratio in chronic obstructive pulmonary disease: a retrospective study. Inflammation. 2014;37(2):374-380.

18. Abakay O, Abakay A, Sen HS, Tanrikulu AC. The relationship between inflammatory marker levels and pulmonary tuberculosis severity. Inflammation. 2015;38(2):691-696.

19. Robinson S, Leonard P. C reactive protein, erythocyte sedimentation rate, or both, in the diagnosis of atraumatic paediatric limb pain? Emerg Med J. 2012;29(12):969-971.

20. Balta S, Demırkol S, Kucuk U. The platelet lymphocyte ratio may be useful inflammatory indicator in clinical practice. Hemodial Int. 2013; 17(4):668-669.

21. Simon R. Sensitivity, specificity, PPV and NPV for predictive biomarkers. J Natl Cancer Inst. 2015;107(8):pii:djv153.

22. Sorensen PD, Jakobsen EH, Madsen JS, et al. Serum HER-2: sensitivity, specificity, and predictive values for detecting metastatic recurrence in breast cancer patients. J Cancer Res Clin Oncol. 2013;139(6): 1005-1013.

23. Malek IA, King A, Sharma H, et al. The sensitivity, specificity and prodictive values of raised plasma metal ion levels in the diagnosis of adverse reaction to metal detris in symptomatic patients with a metaon-metal arthroplasty of the hip. J Bone Joint Surg Br. 2012;94(8): $1045-1050$
International Journal of COPD

\section{Publish your work in this journal}

The International Journal of COPD is an international, peer-reviewed journal of therapeutics and pharmacology focusing on concise rapid reporting of clinical studies and reviews in COPD. Special focus is given to the pathophysiological processes underlying the disease, intervention programs, patient focused education, and self management protocols.
Dovepress

This journal is indexed on PubMed Central, MedLine and CAS. The manuscript management system is completely online and includes a very quick and fair peer-review system, which is all easy to use. Visit http://www.dovepress.com/testimonials.php to read real quotes from published authors. 